\title{
BMJ Open Albuminuria: population epidemiology and concordance in Australian children aged 11-12 years and their parents
}

\author{
Nicholas G Larkins, ${ }^{\oplus, 2,3}$ Siah Kim, ${ }^{1,4}$ John B Carlin, ${ }^{\oplus, 6}$ Anneke C Grobler, ${ }^{\oplus, 7}$ \\ David P Burgner, ${ }^{\circ, 7,8}$ Katherine Lange, ${ }^{\odot 5,7}$ Jonathan C Craig, ${ }^{\odot 1,2}$ \\ Melissa Wake $5,7,9$
}

To cite: Larkins NG, Kim S, Carlin JB, et al. Albuminuria: population epidemiology and concordance in Australian children aged $11-12$ years and their parents. BMJ Open 2019;9:75-84. doi:10.1136/ bmjopen-2017-020262

- Prepublication history and additional material for this paper are available online. To view, please visit the journal (http:// dx.doi.org/10.1136/bmjopen2017-020262).

Received 24 0ctober 2017 Revised 15 February 2018 Accepted 4 April 2019

Check for updates

(C) Author(s) (or their employer(s)) 2019. Re-use permitted under CC BY-NC. No commercial re-use. See rights and permissions. Published by BMJ.

For numbered affiliations see end of article.

Correspondence to Professor Melissa Wake; melissa.wake@mcri.edu.au

\section{ABSTRACT}

Objectives To describe the distribution of albuminuria among Australian children aged 11-12 years and their parents, and assess its intergenerational concordance within parent-child dyads.

Design Population-based cross-sectional study (the Child Health CheckPoint), nested within the Longitudinal Study of Australian Children.

Setting Assessment centres (seven Australian cities and eight regional towns) and home visits across Australia, February 2015 to March 2016.

Participants Of all participating CheckPoint families ( $n=1874), 1557$ children (46.2\% girls) and 1454 parents (85.5\% mothers) provided random urine samples at the visit; samples from menstruating females were excluded. Outcome measures Urine albumin-to-creatinine ratio (ACR) and its components (urine albumin and creatinine concentration); albuminuria was defined as an ACR $\geq 3.4 \mathrm{mg} / \mathrm{mmol}$. Pearson's correlation coefficients and multivariable linear regression models assessed parentchild concordance, using log-transformed data due to skewing. Survey weights and methods were applied to account for the complex sample design.

Results The median ACR for children was $1.03 \mathrm{mg} /$ $\mathrm{mmol}$ (IQR 0.65-1.97) and $1.01 \mathrm{mg} / \mathrm{mmol}$ (IQR 0.60-2.09) for adults. The median ACR was higher in girls (1.20, IQR $0.71-2.65)$ than boys $(0.90$, IQR $0.61-1.65)$ and in mothers $(1.13$, IQR $0.63-2.33)$ than fathers $(0.66$, IQR $0.41-1.05)$. Albuminuria was detected in $15.1 \%$ of children (girls $20.8 \%$, boys $10.1 \%$ ) and $13.5 \%$ of adults $(15.1 \%$ mothers, $4.0 \%$ fathers) had albuminuria. There was a small correlation between parent and child ACR (Pearson correlation coefficient $0.06,95 \% \mathrm{Cl} 0.01$ to 0.12 ).

Conclusions Albuminuria is common among Australian children and adults, which is of concern because it predicts risk for kidney and cardiovascular disease, and mortality. The weak concordance among intergenerational pairs for urine ACR suggests either that genetic heritability is low or that it becomes evident only at later offspring life stages.

\section{INTRODUCTION}

Albuminuria, even at low levels, is an important marker of chronic kidney disease and strongly predicts end-stage kidney disease. ${ }^{12}$ It also independently predicts cardiovascular events

\section{Strengths and limitations of this study}

- Australia-wide population-based cohort sampled using a multistage, clustered survey design allowing for the derivation of accurate population-level estimates.

- Large sample of biological parent-child pairs allowing for precise estimates of intergenerational concordance.

- First morning or timed urine samples were not available to investigate the impact of orthostatic variation on urine albumin-to-creatinine ratio (ACR). The estimates of prevalence would be lower if participants with intermittent proteinuria were identified and excluded.

- The single (rather than repeated) urine ACR value makes the study open to residual measurement error.

and all-cause mortality, probably as a reflection of endothelial dysfunction. ${ }^{3}$ There are different methods of measuring albuminuria. The reference standard, 24-hour urine collection, is impractical in many settings, so that spot urine albumin-to-creatinine ratio (ACR) is routinely used in clinical practice. Urine ACR and spot urine albumin concentration reliably estimate 24-hour albumin excretion, although the correlation is stronger for ACR because it accounts for differences in urine concentration due to hydration status. ${ }^{4}$ There are also postural changes in albumin excretion. ${ }^{5}$ However, because the timing of collection tends not to correlate with explanatory variables of interest (eg, socioeconomic status or age) this does not introduce confounding. Consequently, random samples are routinely used for research purposes also and most evidence relating albuminuria to kidney and cardiovascular risk derives from random urine ACR. ${ }^{6}$

Despite the potential utility of urine ACR as a cheap, non-invasive biomarker, data about the distribution of ACR and its 
components in children are mostly limited to disease-specific contexts. ${ }^{7}$ Thus, its applicability as a marker of risk in well children is unknown. Nonetheless, it has been widely adopted because its ease of collection makes it feasible to bring children's kidney health into population studies. In children with type 1 diabetes mellitus, persistent or intermittent microalbuminuria predicts the future development of macroalbuminuria, and in children with renal dysplasia or glomerular pathology albuminuria is also predictive of long-term renal function. ${ }^{8-12}$ However, the population-based data that are available in children suggest some important discrepancies compared with adults. For example, whereas obese adult have higher rates of albuminuria, the reverse has been reported in children (an OR of 0.34 for albuminuria in overweight and obese compared with normal weight children). ${ }^{13}$ In addition, orthostatic proteinuria, generally considered to be a benign condition, may affect up to $20 \%$ of children 9-16 years old. ${ }^{13-15}$

Among adults, given the clear relationship between albuminuria and future cardiovascular events or chronic kidney disease, there has been a shift in focus from pathological levels to the risk associated with urine ACR levels in the low-normal range. The Chronic Kidney Disease Prognosis Consortium has shown through collaborative meta-analysis that a urine ACR of $1.1 \mathrm{mg} / \mathrm{mmol}$ is associated with a HR of 1.20 for all-cause mortality (95\% CI 1.15 to 1.26 ) compared with a urine ACR of $0.6 \mathrm{mg} / \mathrm{mmol} .{ }^{6}$ Such relationships, where risk extends below traditional thresholds into the normal range, are common among clinical biomarkers and make it difficult to define the threshold at which values should be considered abnormal. ${ }^{1617}$

It is increasingly recognised that the development of chronic diseases, such as kidney and cardiovascular disease, begins in early life. ${ }^{18}$ Through the study of parent-child concordance for early disease markers or risk factors, we can begin to understand the role of inherited genetic and environmental influences in establishing adverse lifecourse trajectories. A substudy of the Framingham Heath Study involving 1055 adult participants found up to $20 \%$ of the population variance in urine ACR may be due to genetic heritability. ${ }^{19}$ Single-nucleotide polymorphism data also suggests a genetic component to albuminuria. ${ }^{20}$ If concordance is confirmed while offspring are still children, this could provide useful prognostic information early in life as we attempt to develop strategies allowing the earlier identification of people progressing towards disease states, with the ultimate goal of providing earlier interventions to keep people healthy.

The Child Health CheckPoint study nested within Growing Up in Australia (also known as the Longitudinal Study of Australian Children, LSAC) provides an important opportunity to examine these issues. We aimed to assess the cross-sectional distribution of urine ACR and related metrics in Australian children aged 11-12 years and their parents, and describe the extent of intergenerational concordance for this measure.

\section{METHODS}

\section{Study design and participants}

Details of the initial LSAC study design and recruitment are outlined elsewhere. ${ }^{21} 22$ In brief, LSAC recruited a nationally representative sample of 5107 infants using a two-stage random sampling design with postcode as the primary sampling unit, and followed them up in biennial 'waves' of data collection up to 2015. The initial proportion recruited to the relevant B cohort in 2004 was $57.2 \%$, of whom $73.7 \% \quad(\mathrm{n}=3764)$ were retained to LSAC wave 6 in 2014.

At the wave 6 visit, all remaining families $(n=3513)$ were invited to consent to their contact details being shared with the Child Health CheckPoint (CheckPoint) team. In 2015, families who consented were then sent an information pack via post and received an information and recruitment phone call. The CheckPoint's detailed cross-sectional biophysical assessment, nested between LSAC waves 6 and 7 (aged 11-12 years), took place between February 2015 and March 2016. One thousand eight hundred and seventy-four families participated. A more detailed description of the CheckPoint study design is available elsewhere. ${ }^{23} 24$

\section{Patient and public involvement}

Because LSAC is a population-based longitudinal study, no patient groups were involved in its design or conduct. To our knowledge, the public was not involved in the study design, recruitment or conduct of the LSAC study or its CheckPoint module. Parents received a summary health report for their child and themselves at or soon after the CheckPoint assessment visit. They consented to take part knowing that they would not otherwise receive individual results about themselves or their child.

\section{Consent}

The attending parent provided written informed consent for themself and their child to participate in the study.

\section{Procedure}

Urine was collected at a specialised 3.5-hour (major and large cities) or 2.5-hour (smaller regional centres) CheckPoint assessment centre visits; those families $(n=378)$ who could not attend were offered a shorter home visit (figure 1). Child Health CheckPoint assessments took place between February 2015 and March 2016. As assessment centre visits ran throughout the day, sample collection time could be anywhere between 09:00 and 18:00, even though urine collection was requested as part of the first assessment station after check-in. Participants were asked to urinate directly into a polypropylene sterile pot until full (or as best possible), and included in current analyses if they provided a usable sample (figure 1). Results from mothers and girls who self-reported they were menstruating were excluded. Only biological parent-child pairs were included for concordance analysis. 


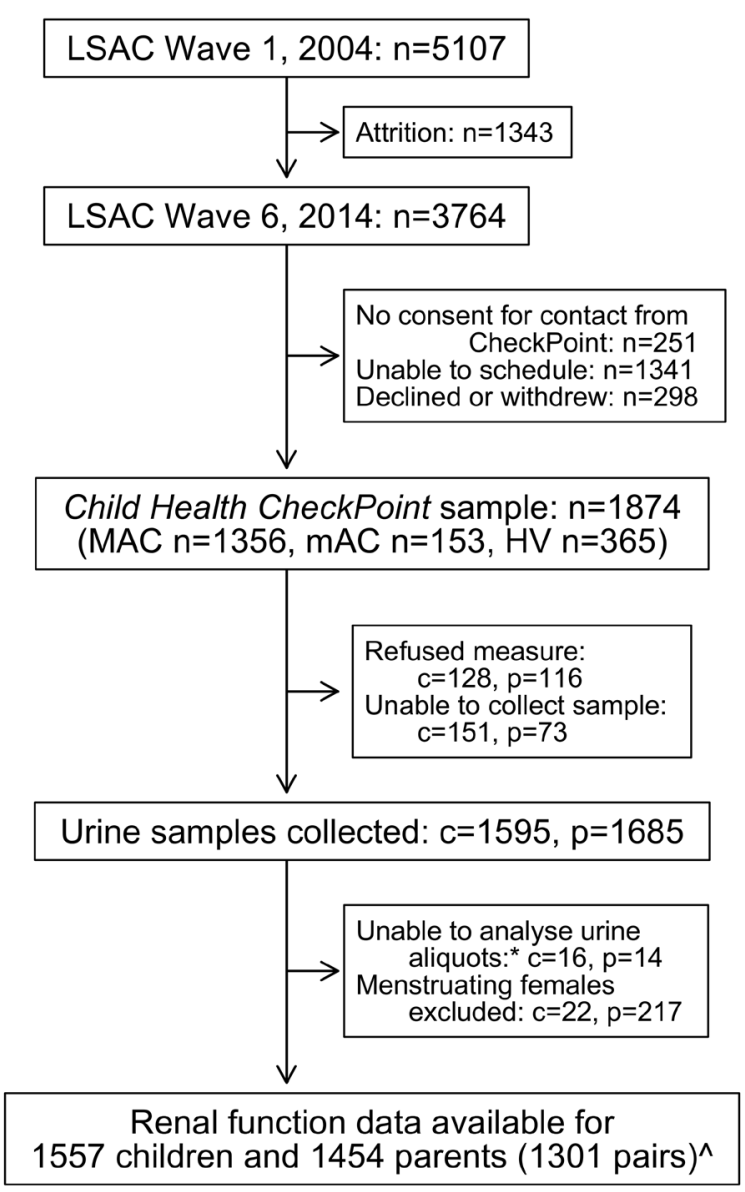

Figure 1 Participant flowchart. ACR, albumin-to-creatinine ratio; c, number of children; HV, home visit assessment; LSAC, Longitudinal Study of Australia Children; MAC, main assessment centre; mAC, miniassessment centre; $\mathrm{n}$, number of families; $\mathrm{p}$, number of attending adults . *Unable to analyse due to insufficient volume of poor quality sample. ^Data excluded from 2 children and 1 parent with ACR $>200 \mathrm{mg} / \mathrm{mmol}$ or albumin $>2000 \mathrm{mg} / \mathrm{L}$, and data from 15 non-biological child-parent pairs excluded from concordance analyses.

\section{Outcome measure}

Urine samples were stored at $4^{\circ} \mathrm{C}$ until processing $(77 \%$ of samples processed within 3 hours and $85 \%$ within 13 hours; maximum 7 days for home visit participants). They were then pipetted under laboratory conditions into a maximum of $12 \times 0.7 \mathrm{~mL}$ aliquots and frozen and stored at $-80^{\circ} \mathrm{C}$. Analysis was performed at the Laboratory of the Baker Heart and Diabetes Institute. Samples were first defrosted over $30 \mathrm{~min}$ and then centrifuged at $500 \mathrm{rpm}$ for $3 \mathrm{~min}$. A Cobas Integra 400 plus analyzer performed the measurements, determining albumin using an immunoturbimetric assay and creatinine using the enzymatic colorimetric method. The lower limit for the detection of urine albumin on this machine was $3 \mathrm{mg} / \mathrm{L}$ and for urine creatinine was $0.1 \mathrm{mmol} / \mathrm{L}$.

For descriptive purposes, ACR was categorised according to the Kidney Disease Improving Global
Outcomes (KDIGO) 2012 guidelines: microalbuminuria $3.4-34 \mathrm{mg} / \mathrm{mmol}$ and macroalbuminuria $>34 \mathrm{mg} /$ mmol. ${ }^{25}$ We also determined the prevalence of albuminuria among adults using the current Australian guidelines: microalbuminuria in fathers $2.5-25 \mathrm{mg} / \mathrm{mmol}$ and macroalbuminuria in fathers $>25 \mathrm{mg} / \mathrm{mmol}$, microalbuminuria in mothers $3.5-35 \mathrm{mg} / \mathrm{mmol}$ and macroalbuminuria in mothers $>35 \mathrm{mg} / \mathrm{mmol} .{ }^{26}$ For purposes of this paper, we used the overarching term 'albuminuria' to refer to the combined participants with microalbuminuria or macroalbuminuria.

\section{Potential confounders \\ Disadvantage score}

In Australia, Socioeconomic Indexes for Areas provide standardised scores for socioeconomic position by geographic area (postcode of family domicile) compiled from 2011 Australian census data. We used the Iindex of Rrelative Ssocioeconomic Ddisadvantage (Disadvantage Index) which numerically summarises the social and economic conditions of Australian neighbourhoods (national mean of 1000 and SD of 100, where higher values represent less disadvantage). ${ }^{27}$

\section{Anthropometry}

Height was measured twice, three times where the first two measures differed by $>0.5 \mathrm{~cm}$, without shoes or socks using a portable rigid stadiometer (model 10955, Invicta, UK), and all measurements averaged to produce the final value. An electronic, calibrated scale (lnBody230 scales, Biospace Co., South Korea) was used to measure body weight with participants wearing light clothing and no shoes or socks. Body mass index (BMI) for children was converted to z-scores according to the 2000 Centers for Disease Control and Prevention growth charts. ${ }^{28}$ Overweight was defined as BMI $\geq 85$ th centile and $<95$ th centile, and obesity as BMI $\geq 95$ th centile. ${ }^{29}$ A steel tape measure was used to measure waist circumference using the crosshand technique at the narrowest point (or mid-point if no narrowing) between then 10th rib and the top of the iliac crest. Two measurements were taken provided these were within $0.1 \mathrm{~cm}$, otherwise a third measurement was performed. The final value was the mean of the two closest measurements.

\section{Blood pressure}

Blood pressure was measured at the brachial artery using an oscillometric device (SphygmoCor XCEL, AtCor Medical Pty., Australia), following at least $7 \mathrm{~min}$ of rest, in the supine position. Three measurements were taken with at least $1 \mathrm{~min}$ of rest between readings. The final value was the average of all three measurements. Hypertension and high-normal blood pressure were defined as a height, sex and age adjusted blood pressure centile $\geq 95$ th centile or $<95$ th but $\geq 90$ th centile, respectively, using the fourth report from the National High Blood Pressure Education Program reference thresholds. ${ }^{30} 31$ 
Other

Medical history was by obtained by self-report or guardian report. Pubertal status was determined using self-reported responses on the Pubertal Development Scale. $^{32}$

\section{Statistical analysis}

Concordance between parents and children was assessed by (1) Pearson's correlation coefficients with 95\% CIs and (2) linear regression with the child variable as dependent variable and parent variable as independent variable. Linear regression models were adjusted for parent age, Disadvantage Index, and parent and child BMI and sex in models including both sexes. In addition, the Pearson's correlation coefficient and linear regression analyses were repeated using weighted multilevel survey analyses. These results were similar to the simple correlations and regression results adjusted for stratification and clustering by postcode only, and only the simple results are reported. Two children and one adult showed an ACR > 200 mg/ mmol or urine albumin concentration $>2000 \mathrm{mg} / \mathrm{L}$. These were included in the distribution statistics consistent with the population-based sampling and aims. However, they were excluded from the concordance analyses because they were outliers and influential observations, considered to represent discrete glomerular pathology.

Population summary statistics and proportions were estimated by applying survey weights and survey procedures that corrected for sampling and participation biases and took into account clustering in the sampling frame. SEs were calculated taking into account the complex design and weights. ${ }^{33}$ More detail on the calculation of weights is provided elsewhere. ${ }^{34}$

The differences between adult and child, and between male and female, groups were tested for statistical significance using linear or logistic regression. All urine variables were log-transformed for the purposes of hypothesis testing and concordance analysis, due to severe right skewing.

\section{RESULTS}

\section{Sample characteristics}

Of the 1874 families assessed, 1557 children (83.1\%) and 1454 adults $(90.0 \%)$ contributed valid urine samples for analysis (1301 biological parent-child pairs; figure 1). Twenty-two children and 217 adults were excluded because they were menstruating, and 15 carer-child pairs were excluded from concordance analysis because the adult was not a biological parent. There were slightly more boys $(n=837,53.8 \%)$ than girls $(n=720)$ and most parents were mothers $(n=1243,85.5 \%)$ (table 1$)$.

The participant characteristics were broadly reflective of the Australian population, as per the study design, with the exception that the sample was relatively less

\begin{tabular}{|c|c|c|c|c|c|c|c|c|c|c|c|c|}
\hline \multirow[b]{2}{*}{ Characteristic } & \multicolumn{3}{|c|}{ Boys } & \multicolumn{3}{|c|}{ Girls } & \multicolumn{3}{|c|}{ Fathers* } & \multicolumn{3}{|c|}{ Mothers* } \\
\hline & $\mathrm{n}$ & $\mathbf{N}$ & $\% \dagger$ & $\mathbf{n}$ & $\mathbf{N}$ & $\% \dagger$ & $\mathbf{n}$ & $\mathbf{N}$ & $\% \dagger$ & $\mathbf{n}$ & $\mathbf{N}$ & $\% \dagger$ \\
\hline Age (years), mean† (SD) & 835 & 12.0 & 0.38 & 720 & 12.0 & 0.42 & 211 & 46.7 & 6.89 & 1243 & 43.5 & 5.35 \\
\hline \multicolumn{13}{|l|}{ BMI category } \\
\hline Overweight & 127 & 836 & 16.3 & 107 & 720 & 16.8 & 90 & 210 & 42.9 & 382 & 1237 & 31.2 \\
\hline Obese & 84 & 836 & 12.7 & 59 & 720 & 9.6 & 71 & 210 & 37.2 & 382 & 1237 & 32.4 \\
\hline \multicolumn{13}{|l|}{ Blood pressure category } \\
\hline High-normal & 18 & 787 & 2.5 & 26 & 692 & 4.1 & 111 & 197 & 57.0 & 372 & 1164 & 32.7 \\
\hline Hypertensive & 24 & 787 & 4.9 & 22 & 692 & 3.6 & 31 & 197 & 18.1 & 88 & 1164 & 7.8 \\
\hline \multicolumn{13}{|l|}{$\begin{array}{l}\text { Socioeconomic status } \\
\text { quintile }\end{array}$} \\
\hline $\begin{array}{l}\text { First (most } \\
\text { disadvantaged) }\end{array}$ & 70 & 833 & 13.4 & 54 & 719 & 10.6 & 17 & 208 & 11.9 & 93 & 1242 & 12.2 \\
\hline Second & 128 & 833 & 19.6 & 107 & 719 & 17.9 & 37 & 208 & 22.6 & 181 & 1242 & 17.6 \\
\hline Third & 145 & 833 & 19.8 & 134 & 719 & 20.6 & 40 & 208 & 23.3 & 225 & 1242 & 20.3 \\
\hline Fourth & 194 & 833 & 21.8 & 179 & 719 & 25.1 & 37 & 208 & 15.8 & 317 & 1242 & 24.8 \\
\hline Fifth (least disadvantaged) & 296 & 833 & 25.5 & 245 & 719 & 25.8 & 77 & 208 & 26.4 & 426 & 1242 & 25.1 \\
\hline Diabetes & 2 & 837 & 0.2 & 3 & 720 & 0.6 & 9 & 211 & 4.8 & 26 & 1243 & 2.6 \\
\hline Heart condition & - & - & - & - & - & - & 9 & 211 & 5.4 & 24 & 1243 & 2.7 \\
\hline Started puberty & 686 & 793 & 87.8 & 629 & 663 & 94.8 & - & - & - & - & - & - \\
\hline
\end{tabular}

${ }^{*} 98.9 \%$ of adult participants were biological parents of participating children.

†Weighted mean/percentage.

$\mathrm{BMI}$, body mass index; $\mathrm{N}$, number with valid data for characteristic (denominator). 
Table 2 Distribution of urine albumin, creatinine, albumin-to-creatinine ratio and albuminuria by age cohort and sex

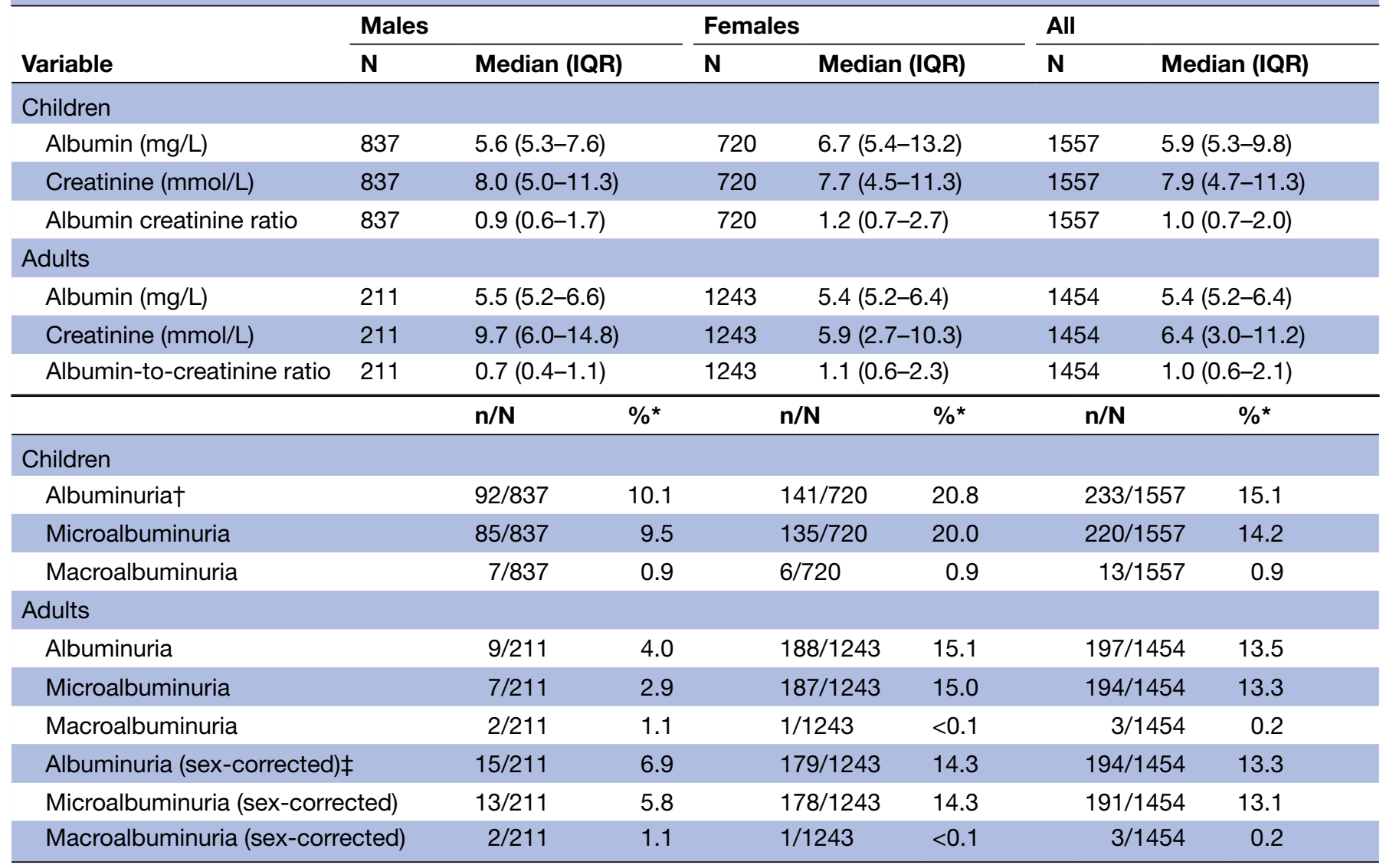

*Weighted percentage.

†Combined total of microalbuminuria and macroalbuminuria.

fUsing current Australian thresholds to define albuminuria, $2.5 \mathrm{mg} / \mathrm{mmol}$ for fathers and $3.5 \mathrm{mg} / \mathrm{mmol}$ for mothers.

$\mathrm{N}$, number with valid data for characteristic (denominator).

disadvantaged than the general population $(25.6 \%$ of children in the least but only $12.1 \%$ in the most disadvantaged national quintile). Consistent with this, most adults and over one-quarter of children were overweight or obese $(65.9 \%$ adults, $27.8 \%$ children). There were no major differences in participant characteristics by sex for the childhood cohort. However, mothers were on average younger than fathers (43.5 vs 46.7 years), fewer had a high-normal BP or hypertension $(40.5 \%$ vs $75.1 \%)$ and fewer were overweight or obese $(63.6 \%$ vs $80.1 \%)$.

\section{Distribution of ACR and albuminuria}

Children

The median ACR among children was $1.03 \mathrm{mg} / \mathrm{mmol}$ (IQR 0.65-1.97) (table 2).

The median urine albumin concentration for boys was $5.6 \mathrm{mg} / \mathrm{L}$ (IQR 5.3-7.6) and for girls was $6.7 \mathrm{mg} / \mathrm{L} \mathrm{(IQR}$ 5.4-13.2; $\mathrm{p}<0.001)$. Albuminuria was present in $15.1 \%$ of children, and albuminuria was more common in girls than boys $(20.8 \%$ vs $10.1 \%$; $\mathrm{p}<0.001)$, largely due to differences in urine albumin as opposed to urine creatinine concentration (figure 2). Mean (SD) data, and log-transformed data are presented in online supplementary table S1. Percentile values for urine albumin, creatine and
ACR distributions are presented in online supplementary tables S2a and S2b.

Adults

The median ACR among adults was $1.01 \mathrm{mg} / \mathrm{mmol}$ (IQR $0.60-2.09)$. The prevalence of albuminuria was much higher in mothers than fathers, at $15.1 \%$ versus $4.0 \%$, respectively $(\mathrm{p}<0.001)$ for the KDIGO thresholds, and $14.3 \%$ versus $6.9 \%$, respectively $(\mathrm{p}=0.02)$ for the sex-stratified Australia thresholds, noting that the number of fathers on which this estimate was based was small. Among adults, the sex discrepancy in ACR was driven more by differences in the distribution of urine creatinine than urine albumin concentration (figure 2).

\section{Intergenerational concordance of ACR}

A weak positive correlation was present between biological parent and child urine ACR ( $\mathrm{r} 0.06,95 \%$ CI 0.01 to 0.12 ) and albumin ( $\mathrm{r} 0.06,95 \% \mathrm{CI} 0.01$ to 0.12 ), with stronger correlations for creatinine values ( $\mathrm{r} 0.19,95 \%$ CI 0.13 to 0.24 ) (figure 3 ). These values were essentially unchanged when using partial correlation coefficients to adjust for parent age, socioeconomic status, and child and parent BMI and sex (table 3, sex-specific values shown in 

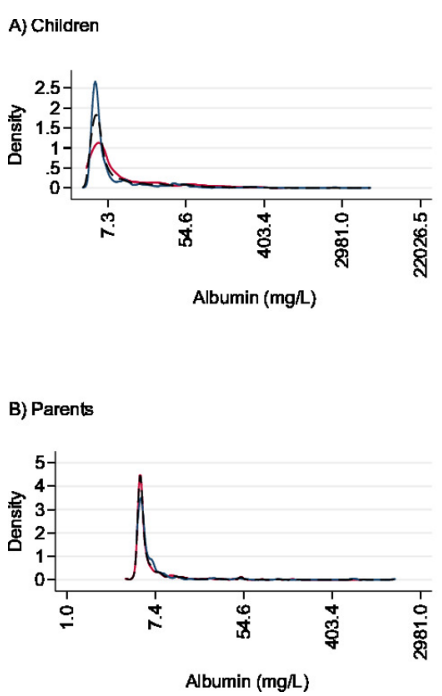
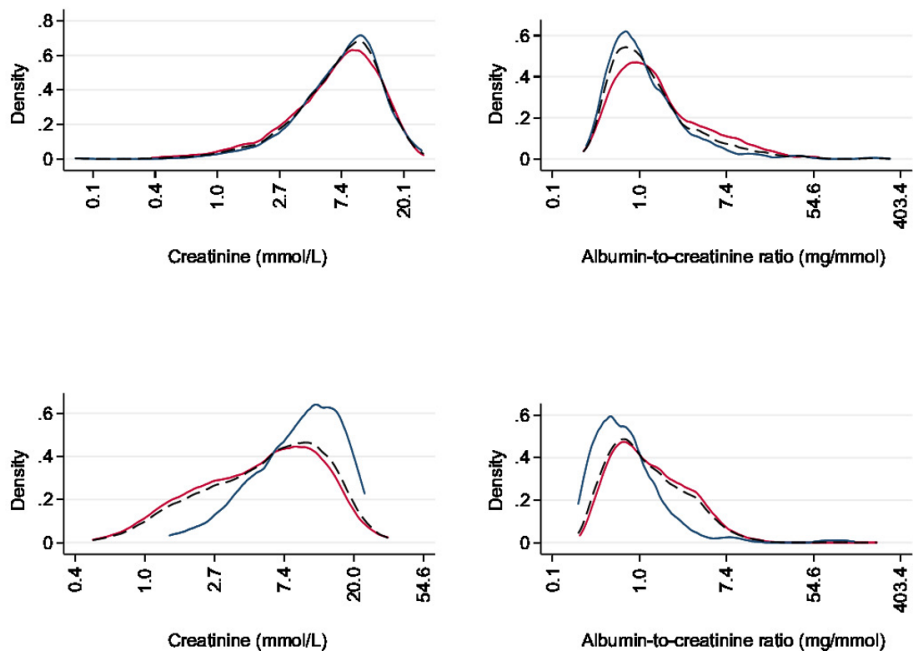

Figure 2 Density plots for urine measures. Red line: girls/mothers; blue line: boys/fathers; black, dotted line: all. Graphs plot log-transformed data, with $\mathrm{x}$-axis labelled using actual values for ease of interpretation.

online supplementary tables S3a and S3b). Multivariable linear regression showed some evidence of parent-child concordance for all three urine variables (table 3).

There was also a weak association between parent-child pairs for albuminuria (ACR $\geq 3.4 \mathrm{mg} / \mathrm{mmol}$ ): in $73.3 \%$ of pairs neither parent or child had albuminuria, in $11.8 \%$ of pairs only the adult had albuminuria, in $12.8 \%$ of pairs only the child had albuminuria and in $2.0 \%$ both parent and child had albuminuria.

\section{DISCUSSION}

\section{Principal findings}

In this population-based Australian study, $13.5 \%$ of adults and $15.1 \%$ of children had albuminuria. The prevalence varied by sex, with albuminuria more common in females among both children and, especially, adults. In children, the sex difference was mostly driven by an increased urine albumin concentration, whereas in adults the urine creatinine concentration was relatively more important. There was weak intergenerational concordance for urine albumin and ACR, but creatinine concordance was larger (0.15-0.19).

\section{Strengths and limitations}

Strengths of the Child Health CheckPoint study include the large sample size and the multistage, clustered survey methodology that together allow for the derivation of accurate population estimates. All samples were processed in a central research laboratory under strict protocols. The main weakness of the data presented here is that first morning or timed samples were not collected, and so we cannot account for the impact of orthostatic proteinuria. We also lacked the ability to account for day-to-day variability in urine ACR, which will result in residual measurement error. ${ }^{35}$ Orthostatic proteinuria is a greater issue for children than adults and may have increased the prevalence estimates. ${ }^{36}$ Sample attrition is a common limitation among long-running longitudinal studies. Retention in LSAC was similar to other birth cohorts at this age. ${ }^{37} 38$ Not all remaining families participated in CheckPoint. However, the sample remained similar to of the Australian population in most respects and survey weighting was used to account for drop-out and non-response among children. ${ }^{23}$ There was an over-representation of mothers in the adult cohort, reducing the precision around estimates for fathers. The lack of longitudinal biospecimen data limits our ability to draw conclusions about the prevalence of albuminuria over time or investigate how this relates to future events.

\section{Findings in relation to other studies}

The prevalence of albuminuria among children was similar to other population-based studies. We recently performed an analysis of data from the 2011/2013 Australian Health Survey (AHS) and found $12.8 \%$ of children aged 5-18 years had albuminuria using the same threshold of $3.4 \mathrm{mg} / \mathrm{mmol}^{13}$ In the USA, the prevalence of albuminuria in the National Health and Nutrition Examination Survey (NHANES) has been reported as $8.9 \% .{ }^{14}$ However, in that study the mean age was 15.5 years, and older adolescents appear to have a lower prevalence of albuminuria. ${ }^{139}$ In both the AHS and NHANES studies, girls had a higher ACR than boys because of differences in urine albumin, rather than differences in urine creatinine concentration. We believe the most likely explanation for this is that normal physiological values vary by sex, rather than sex-related differences in cardiovascular or kidney health at this age.

The prevalence of albuminuria and mean values for urine ACR, urine albumin and urine creatinine concentrations were similar in children and adults. This was unexpected because, as a marker of early disease and risk for future clinical cardiovascular and kidney events, we would expect an increase in the prevalence 

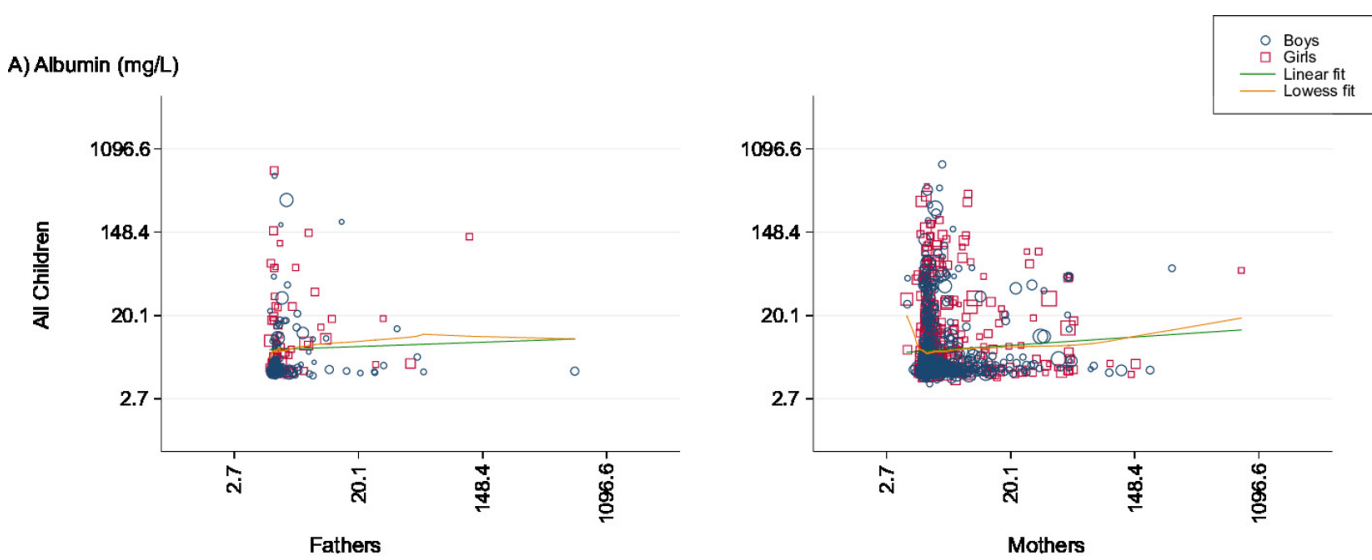

B) Creatinine (mmol/L)
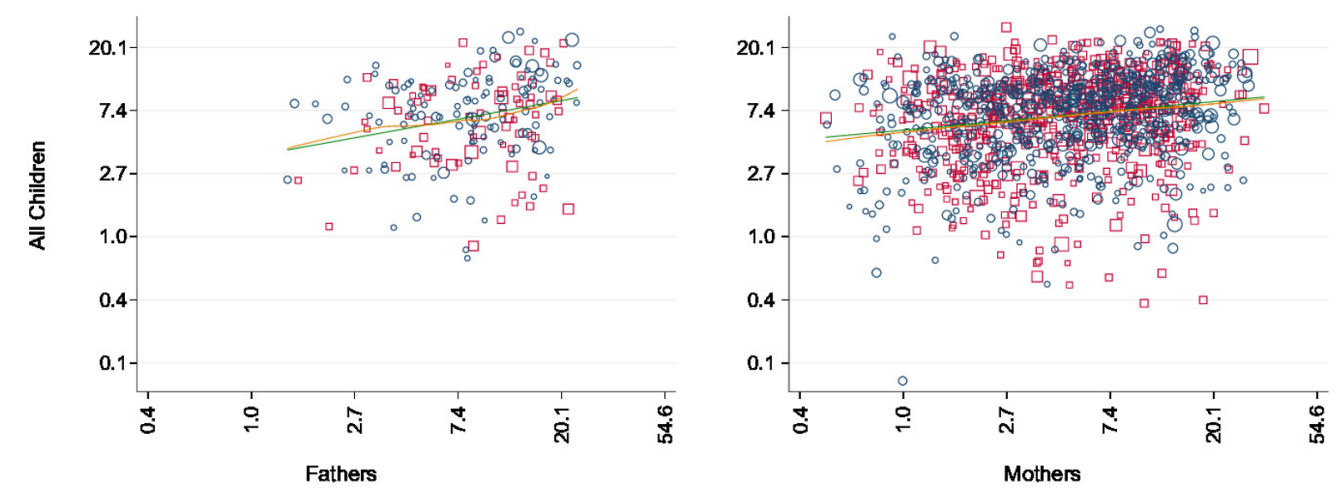

C) Albumin-to-creatinine ratio ( $\mathrm{mg} / \mathrm{mmol})$
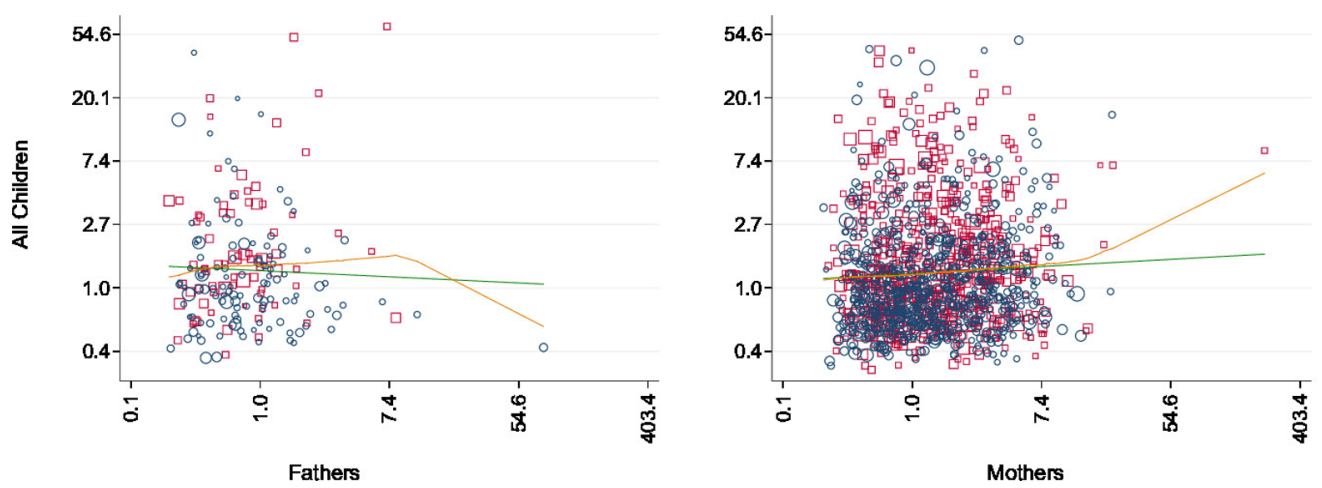

Figure 3 Parent-child pair scatter plots. Graphs plot log-transformed data, with x-axis labelled using actual values for ease of interpretation.

of albuminuria between childhood and mid-adulthood, as is observed between young and older adults. ${ }^{40}$ Similar to the observed sex discrepancy, we suspect this observation is also partly due to a normal physiological variation. There are some reported differences in albumin excretion between children and adults. For example, while overweight and obesity are likely to lead to an increasing prevalence of kidney and cardiovascular disease in later life, in childhood they are paradoxically associated with a lower urine ACR. ${ }^{13} 14$ This is in keeping with other counter-intuitive relationships at this age, for example, obese children showing apparently better flow-mediated dilatation. ${ }^{41}$ There is also some evidence to suggest that children have a greater orthostatic variation in albumin excretion than adults. ${ }^{36}$ In addition to physiological factors, there may be a pathological component to the high urine ACR levels observed among some children. These two components will be difficult to delineate until there are follow-up data on the persistence or regression of albuminuria when participants reach adulthood, and ideally data on the cumulative incidence of downstream clinical events.

Nonetheless, the adult participants in our study had a higher prevalence of albuminuria than anticipated. This was driven mostly by a high prevalence of albuminuria among mothers, who comprised $85.5 \%$ of the adult cohort. While it is well established that average ACR values are higher among adult females, this does 


\begin{tabular}{llll}
\hline Table 3 & Parent-child concordance & & \\
\hline Variable & & & \\
\hline Pearson's correlation & $\mathrm{N}$ & $\mathrm{CC}$ & $95 \% \mathrm{Cl}$ \\
\hline Albumin (mg/L) & 1300 & 0.06 & 0.01 to 0.11 \\
Creatinine (mmol/L) & 1301 & 0.19 & 0.13 to 0.24 \\
Albumin-to-creatinine ratio & 1300 & 0.06 & 0.01 to 0.12 \\
Multivariable linear regression* & $\mathrm{N}$ & $\mathrm{RC}$ & $\mathrm{P}$ value \\
Albumin (mg/L) & 1292 & 0.09 & 0.06 \\
Creatinine (mmol/L) & 1293 & 0.15 & $<0.001$ \\
Albumin-to-creatinine ratio & 1292 & 0.07 & 0.02 \\
Partial correlation coefficient & $\mathrm{N}$ & $\mathrm{RC}$ & \\
Albumin (mg/L) & 1292 & 0.05 & \\
Creatinine (mmol/L) & 1293 & 0.18 & \\
Albumin-to-creatinine ratio & 1292 & 0.07 & \\
\hline
\end{tabular}

*Adjusted for parent age, index of relative socioeconomic disadvantage, child and parent body mass index, parent and child sex. Albumin, creatinine and albumin-to-creatinine ratio have been log transformed.

CC, Pearson's correlation coefficient; RC, estimated regression coefficient.

not always translate into a higher prevalence of albuminuria. ${ }^{42}$ In our study, the main reason for the observed sex discrepancy in ACR was a lower median urine creatinine concentration among mothers, which explains their higher prevalence of albuminuria despite a slightly lower prevalence of risk factors such as hypertension and obesity. ${ }^{43}$ Compared with our overall value of $13.5 \%$, the 2011/2013 AHS reported a prevalence of albuminuria $<10 \%$ until the age of 65 years (using sex-adjusted thresholds), ${ }^{40}$ and the earlier AusDiab study found a lower prevalence again $(6.6 \%) \cdot{ }^{43}$ Overall, it does seem that the prevalence of albuminuria among Australians is increasing over time, consistent with Australians' increasing BMI ${ }^{40} 43$ and with a lower 2008 prevalence of $8.1 \%$ in the USA on a single, random sample. ${ }^{44}$

The parent-child concordance in albumin and ACR were low, consistent with genomic data regarding the heritability of ACR. ${ }^{19}{ }^{20}$ Shared environmental risk factors are also likely to have contributed to this observation. It may be that familial concordance is only unmasked at older ages, in keeping with the higher familial clustering of albuminuria observed among sibling adults in the Framingham study. ${ }^{19}$ For children, this weak intergenerational concordance suggests that family history in itself will be insufficient to identify at-risk individuals and that additional risk factors will need to be considered if and when developing screening and intervention strategies.

\section{Meaning and implications for clinicians and policymakers}

Our results have implications for the determination of albuminuria thresholds in children and adults. For children, thresholds may need to be increased and sex stratified for random, single measurement, spot urine ACR. The current KDIGO guidelines acknowledge that the exclusion of patient-specific factors in defining albuminuria is controversial, but argue an ACR of $3.4 \mathrm{mg} /$ $\mathrm{mmol}$ is more than three times the normal value $(1 \mathrm{mg} /$ $\mathrm{mmol}$ ) in young adults and tied to risk regardless of age, sex and ethnicity. Our data suggest that an ACR of $3.4 \mathrm{mg} / \mathrm{mmol}$ lies within the normal range for children, and there are also no data tying childhood ACR to future clinical events. Thus, the logic behind the exclusion of patient-specific factors cannot be applied to children and the use of a single threshold across all ages needs to be further explored. Repeated, first morning and/or timed samples may be necessary to accurately separate children at-risk of future kidney and cardiovascular events from those with physiologically higher ACR. However, this must be balanced against the risk of excluding kidney markers from large population studies for which feasibility is paramount.

For the adult cohort of CheckPoint, it is hard to reconcile a higher prevalence of albuminuria among mothers (here driven largely by a lower urine creatinine concentration) when females on average have a lower risk of cardiovascular and kidney disease at any given age. ${ }^{45} 46$ This suggests limitations in the thresholds currently used to define albuminuria, which have been derived based on the average urine ACR value required to meet the definition of an abnormal albumin excretion rate. ${ }^{26}$ While this might appear to be a reasonable approach, the definition of an abnormal albumin excretion rate is based on limited distribution data and studies relating albumin excretion to the future development of diabetic nephropathy as defined by the development of a positive urine dipstick result. ${ }^{47-51}$ New ACR thresholds could perhaps be defined by such data directly relating ACR to the risk of cardiovascular or kidney disease. However, this would increase the numbers identified as having albuminuria, thus if applied to clinical practice this runs the risk of generating overdiagnosis in people who are unlikely to benefit from treatment where trial data are limited, as is the case here. ${ }^{161752}$ It is also important to remember that a constant relative risk for a biomarker across its normal range does not equate to a constant risk difference, and there may not be a clinically meaningful increase in events or net benefit to treatment at lower levels. ${ }^{17}$

\section{Unanswered questions and future research}

In summary, the prevalence of albuminuria among adult Australians is concerning with regards to their risk of future cardiovascular and kidney disease. Given this, and that specific treatments already exist (ACE inhibitors and angiotensin receptor blockers), we need trials to determine if the treatment of isolated albuminuria is beneficial, at what threshold, and if treatment targets for patients with concomitant hypertension should include urine ACR. Albuminuria is common among Australian children as determined by the measurement of urine ACR on randomly timed, spot urine collection. However, more research is required into threshold selection for 
children and this requires prospective data tying urine ACR to future clinical events.

Author affiliations

${ }^{1}$ Centre for Kidney Research, The Children's Hospital at Westmead, Westmead, New South Wales, Australia

${ }^{2}$ School of Public Health, University of Sydney, Sydney, New South Wales, Australia ${ }^{3}$ Department of Nephrology, Perth Children's Hospital, Nedlands, Western Australia, Australia

${ }^{4}$ School of Women's and Children's Health, University of New South Wales, Sydney, New South Wales, Australia

${ }^{5}$ Murdoch Children's Research Institute, Parkville, Victoria, Australia

${ }^{6}$ Melbourne School of Population and Global Health, University of Melbourne,

Parkville, Victoria, Australia

${ }^{7}$ Department of Paediatrics, University of Melbourne, Parkville, Victoria, Australia

${ }^{8}$ Department of Paediatrics, Monash University, Melbourne, Victoria, Australia

${ }^{9}$ Department of Paediatrics and The Liggins Institute, The University of Auckland, Auckland, New Zealand

Acknowledgements This paper uses unit record data from Growing Up in Australia, the Longitudinal Study of Australian Children. The study is conducted in partnership between the Department of Social Services (DSS), the Australian Institute of Family Studies (AIFS) and the Australian Bureau of Statistics (ABS). REDCap (Research Electronic Data Capture) electronic data capture tools were used in this study. More information about this software can be found at: www.projectredcap.org . The authors thank the LSAC and CheckPoint study participants, staff and students for their contributions.

Contributors NGL, SK, JBC, ACG, DPB, KL, MW and JCC provided critical input into the design of this component of the Child Health CheckPoint study, analysis and interpretation of the study data and manuscript preparation. NGL wrote the first draft of the manuscript. ACG performed the statistical analysis. MW is the principal investigator of the Child Health CheckPoint.

Funding This work was supported by the National Health and Medical Research Council (NHMRC) of Australia (Project Grants 1041352, 1109355), The Royal Children's Hospital Foundation (2014-241), the Murdoch Children's Research Institute (MCRI), the Department of Social Services (DSS), The University of Melbourne, the National Heart Foundation of Australia (100660) and the Financial Markets Foundation for Children (2014-055, 2016-310). The urinary albumin and creatinine quantification was funded through NHMRC Program Grant 633003 Screening and Test Evaluation Program. The following authors were supported by the NHMRC: Senior Research Fellowships to MW (1046518) and DPB (1064629); Postgraduate Research Scholarship to NGL (GNT1114218). The following authors were supported by the National Heart Foundation of Australia: Honorary Future Leader Fellowship to DPB (100369). MW was supported by Cure Kids New Zealand. The MCRI administered the research grants for the study and provided infrastructural support (IT and biospecimen management) to its staff and the study, but played no role in the conduct or analysis of the trial. DSS played a role in study design; however, no other funding bodies had a role in the study design and conduct; data collection, management, analysis and interpretation; preparation, review or approval of the manuscript; and decision to submit the manuscript for publication. Research at the MCRI is supported by the Victorian Government's Operational Infrastructure Support Program.

Disclaimer The findings and views reported in this paper are those of the author and should not be attributed to DSS, AIFS or the ABS.

Competing interests All authors have completed the ICMJE uniform disclosure format and declare financial support for the submitted work from the National Health and Medical Research Council of Australia (NHMRC), The Royal Children's Hospital Foundation, the Murdoch Children's Research Institute (MCRI), The University of Melbourne, the National Heart Foundation of Australia, Financial Markets Foundation for Children and the Victoria Deaf Education Institute. Personal fees were received by MW from the Australian Department of Social Services. MW, NGL and DPB are supported by the NHMRC; DPB by the National Heart Foundation of Australia; and MW by Cure Kids New Zealand. MW received grants from NZ Ministry of Business, Innovation \& Employment and A Better Start/Cure Kids New Zealand, and support from Sandoz to present at a symposium outside the submitted work.

Patient consent for publication Not required.
Ethics approval The CheckPoint study protocol was approved by The Royal Children's Hospital Melbourne Human Research Ethics Committee (33225D) and Australian Institute of Family Studies Ethics Committee (14-26).

Provenance and peer review Not commissioned; externally peer reviewed.

Data sharing statement The Longitudinal Study of Australian Children datasets and technical documents are available to researchers at no cost via a licence agreement. Data access requests are co-ordinated by the National Centre for Longitudinal Data. More information is available at https://dataverse.ada.edu.au/ dataverse/lsac.

Open access This is an open access article distributed in accordance with the Creative Commons Attribution Non Commercial (CC BY-NC 4.0) license, which permits others to distribute, remix, adapt, build upon this work non-commercially, and license their derivative works on different terms, provided the original work is properly cited, appropriate credit is given, any changes made indicated, and the use is non-commercial. See: http://creativecommons.org/licenses/by-nc/4.0/.

\section{REFERENCES}

1. Iseki K, Kinjo K, Iseki C, et al. Relationship between predicted creatinine clearance and proteinuria and the risk of developing ESRD in Okinawa, Japan. Am J Kidney Dis 2004;44:806-14.

2. Verhave JC, Gansevoort RT, Hillege HL, et al. An elevated urinary albumin excretion predicts de novo development of renal function impairment in the general population. Kidney Int Suppl 2004;92:S18-21.

3. Gerstein HC, Mann JF, Yi Q, et al. Albuminuria and risk of cardiovascular events, death, and heart failure in diabetic and nondiabetic individuals. JAMA 2001;286:421-21.

4. Dyer AR, Greenland P, Elliott $P$, et al. Evaluation of measures of urinary albumin excretion in epidemiologic studies. Am J Epidemiol 2004;160:1122-31.

5. Witte EC, Lambers Heerspink HJ, de Zeeuw D, et al. First morning voids are more reliable than spot urine samples to assess microalbuminuria. J Am Soc Nephrol 2009;20:436-43.

6. Matsushita K, van der Velde M, Astor BC, et al. Association of estimated glomerular filtration rate and albuminuria with all-cause and cardiovascular mortality in general population cohorts: a collaborative meta-analysis. Lancet 2010;375:2073-81.

7. Rademacher ER, Sinaiko AR. Albuminuria in children. Curr Opin Nephrol Hypertens 2009;18:246-51.

8. Amin R, Widmer B, Prevost AT, et al. Risk of microalbuminuria and progression to macroalbuminuria in a cohort with childhood onset type 1 diabetes: prospective observational study. BMJ 2008;336:697-701.

9. González Celedón C, Bitsori M, Tullus K. Progression of chronic renal failure in children with dysplastic kidneys. Pediatr Nephrol 2007;22:1014-20.

10. Sanna-Cherchi $S$, Ravani $P$, Corbani V, et al. Renal outcome in patients with congenital anomalies of the kidney and urinary tract. Kidney Int 2009;76:528-33.

11. Coppo R, Lofaro D, Camilla RR, et al. Risk factors for progression in children and young adults with IgA nephropathy: an analysis of 261 cases from the VALIGA European cohort. Pediatr Nephrol 2017;32.

12. Gipson DS, Chin H, Presler TP, et al. Differential risk of remission and ESRD in childhood FSGS. Pediatr Nephrol 2006;21:344-9.

13. Larkins $\mathrm{N}$, Teixeira-Pinto $\mathrm{A}$, Craig J. The population-based prevalence of albuminuria in children. Pediatr Nephrol 2017;32:2303-9.

14. Nguyen S, McCulloch $C$, Brakeman $P$, et al. Being overweight modifies the association between cardiovascular risk factors and microalbuminuria in adolescents. Pediatrics 2008:121:37-45.

15. Murakami M, Hayakawa M, Yanagihara T, et al. Proteinuria screening for children. Kidney Int 2005;67:S23-S27.

16. Moynihan R, Doust J, Henry D. Preventing overdiagnosis: how to stop harming the healthy. BMJ 2012;344:e3502-e02.

17. Doust J, Vandvik PO, Qaseem A, et al. Guidance for modifying the definition of diseases: a checklist. JAMA Intern Med 2017;177:1020-5.

18. Olsen MH, Angell SY, Asma S, et al. A call to action and a lifecourse strategy to address the global burden of raised blood pressure on current and future generations: the Lancet Commission on hypertension. Lancet 2016;388:2665-712.

19. Fox CS, Yang Q, Guo CY, et al. Genome-wide linkage analysis to urinary microalbuminuria in a community-based sample: the Framingham Heart Study. Kidney Int 2005;67:70-4. 
20. Marrachelli VG, Monleon D, Rentero P, et al. Genomic and metabolomic profile associated to microalbuminuria. PLoS One 2014;9:e98227

21. Sanson A, Nicholson J, Ungerer J, et al. Introducing the Longitudinal Study of Australian Children - LSAC Discussion Paper No.1. Melbourne: Australian Institute of Family Studies, 2002.

22. Edwards B. Growing Up in Australia: the Longitudinal Study of Australian Children: Entering adolescence and becoming a young adult. Family Matters 2014;95:5-14

23. Wake M, Clifford S, York E, et al. Introducing Growing Up in Australia's Child Health CheckPoint: A physical health and biomarkers module for the Longitudinal Study of Australian Children. Family Matters 2014 95:15-23.

24. Clifford S, Davies S, Wake M. Child Health CheckPoint: Cohort summary and methodology of a physical health and biospecimen module for the Longitudinal Study of Australian Children. BMJ Open 2019;9(suppl 3):3-22.

25. Stevens PE, Levin A. for the Kidney Disease: Improving Global Outcomes Chronic Kidney Disease Guideline Development Work Group. Evaluation and management of chronic kidney disease: Synopsis of the kidney disease: improving global outcomes 2012 clinical practice guideline. Ann Intern Med 2013;158:825-30.

26. Johnson DW, Jones GR, Mathew TH, et al. Chronic kidney disease and measurement of albuminuria or proteinuria: a position statement. Med J Aust 2012;197:224-5.

27. Australian Bureau of Statistics (ABS). Socio-Economic Indexes for Areas (SEIFA) - Technical Paper 2006. Canberra: ABS, 2008.

28. Ogden CL, Kuczmarski RJ, Flegal KM, et al. Centers for Disease Control and Prevention 2000 growth charts for the United States: improvements to the 1977 National Center for Health Statistics version. Pediatrics 2002;109:45-60.

29. Barlow SE. Expert Committee. Expert committee recommendations regarding the prevention, assessment, and treatment of child and adolescent overweight and obesity: summary report. Pediatrics 2007;120(Suppl 4):S164-S192.

30. Village EG, Falkner B, Daniels SR, et al. The fourth report on the diagnosis, evaluation, and treatment of high blood pressure in children and adolescents. Pediatrics 2004;114:555-76.

31. Lurbe E, Agabiti-Rosei E, Cruickshank JK, et al. European Society of Hypertension guidelines for the management of high blood pressure in children and adolescents. J Hypertens 2016;2016:1887-920.

32. Bond L, Clements J, Bertalli N, et al. A comparison of self-reported puberty using the Pubertal Development Scale and the Sexual Maturation Scale in a school-based epidemiologic survey. J Adolesc 2006;29:709-20.

33. Heeringa SG, West BT, Berglund PA. Applied survey data analysis. Boca Raton, FL: CRC Press, 2010.

34. Ellul S, Hiscock R, Mensah FK, et al. Longitudinal Study of Australian Children's Child Health CheckPoint Technical Paper 1: Weighting and Non-Response. Melbourne: Murdoch Children's Research Institute, 2018

35. Naresh CN, Hayen A, Weening A, et al. Day-to-day variability in spot urine albumin-creatinine ratio. Am J Kidney Dis 2013;62:1095-101.
36. Brandt JR, Jacobs A, Raissy $\mathrm{HH}$, et al. Orthostatic proteinuria and the spectrum of diurnal variability of urinary protein excretion in healthy children. Pediatr Nephrol 2010;25:1131-7.

37. Straker L, Mountain J, Jacques A, et al. Cohort profile: the Western Australian Pregnancy Cohort (Raine) Study-Generation 2. Int J Epidemiol 2017;46:dyw308-85

38. Boyd A, Golding J, Macleod J, et al. Cohort Profile: the 'Children of the 90s'--the index offspring of the Avon Longitudinal Study of Parents and Children. Int J Epidemiol 2013;42:111-27.

39. Sanchez-Bayle M, Rodriguez-Cimadevilla C, Asensio C, et al. Urinary albumin excretion in Spanish children. Pediatric Nephrology 1995;9:428-30.

40. Australian Bureau of Statistics (ABS). Australian Health Survey: Biomedical Results for Chronic Diseases, 2011-12. Canberra: ABS, 2013.

41. Donald AE, Charakida M, Falaschetti E, et al. Determinants of vascular phenotype in a large childhood population: the Avon Longitudinal Study of Parents and Children (ALSPAC). Eur Heart J 2010;31:1502-10.

42. Nitsch D, Grams M, Sang Y, et al. Associations of estimated glomerular filtration rate and albuminuria with mortality and renal failure by sex: a meta-analysis. BMJ 2013;346:f324.

43. Atkins RC, Polkinghorne KR, Briganti EM, et al. Prevalence of albuminuria in Australia: the AusDiab Kidney Study. Kidney Int Suppl 2004;66:S22-S24.

44. Coresh J, Selvin E, Stevens LA, et al. Prevalence of chronic kidney disease in the United States. JAMA 2007;298:2038-47.

45. GBD 2013 Mortality and Causes of Death Collaborators. Global, regional, and national age-sex specific all-cause and cause-specific mortality for 240 causes of death, 1990-2013: a systematic analysis for the Global Burden of Disease Study 2013. Lancet 2015;385:117-71.

46. Clayton P, Hurst K. ANZDATA Registry Report 2016. Adelaide: Australia and New Zealand Dialysis and Transplant Registry, 2016.

47. Viberti GC, Hill RD, Jarrett RJ, et al. Microalbuminuria as a predictor of clinical nephropathy in insulin-dependent diabetes mellitus. Lancet 1982;1:1430-2.

48. Parving $\mathrm{HH}$, Mogensen $\mathrm{CE}$, Jensen $\mathrm{HA}$, et al. Increased urinary albumin-excretion rate in benign essential hypertension. Lancet 1974;1:1190-2.

49. Hutchison AS, O'Reilly DS, MacCuish AC, et al. Albumin excretion rate, albumin concentration, and albumin/creatinine ratio compared for screening diabetics for slight albuminuria. Clin Chem 1988;34:2019-21.

50. Bakker AJ. Detection of microalbuminuria. Receiver operating characteristic curve analysis favors albumin-to-creatinine ratio over albumin concentration. Diabetes Care 1999;22:307-13.

51. Houlihan CA, Tsalamandris C, Akdeniz A, et al. Albumin to creatinine ratio: a screening test with limitations. Am J Kidney Dis 2002;39:1183-9.

52. Brownlee S, Chalkidou K, Doust J, et al. Evidence for overuse of medical services around the world. Lancet 2017;390:156-68. 